\title{
Purificação de resíduos de oficina de artesanato em pedra-sabão por flotação e alvejamento químico
}

\author{
Purification of the soapstone residues, \\ produced in artesan workshops, \\ by flotation and chemical leaching
}

Michael Leonardo Marques Rodrigues

Químico Industrial, aluno do Programa de Pós-Graduação em Engenharia Mineral DEMIN/ UFOP

mitchel.marques@yahoo.com.br

Rosa Malena Fernandes Lima

Professora do Departamento de

Engenharia de Minas da UFOP

rosa@demin.ufop.br

\section{Resumo}

Na região de Ouro Preto-MG, existem diversas oficinas de artesanato em pedra-sabão. Nessas oficinas, o processo de fabricação de panelas e objetos de adorno, produz uma grande quantidade de resíduos, que, normalmente, são depositados nas proximidades das oficinas, sem nenhuma forma de controle. Esse trabalho teve por objetivo recuperar o talco contido na fração granulométrica abaixo de $74 \mu \mathrm{m}$ desses resíduos através de purificação por flotação e alvejamento químico com $\mathrm{HCl}$. Entre as variáveis estudadas na flotação, apenas a porcentagem de sólidos e a dosagem de espumante se mostraram significativas com relação às variáveis-respostas: recuperação mássica e alvura. Nesse processo, produziu-se um concentrado com alvura (ISO) de 68,56\% e com composição química que está de acordo com as especificações de tinta (espalhador) e papel (carga). Após alvejamento químico do concentrado de flotação, foram obtidos alvura (ISO) de 84,0\% e teores químicos que estão dentro das especificações da indústria de plásticos (carga e reforço) e plásticos antiaderentes.

Palavras-chave: Pedra-sabão, talco, resíduos.

\begin{abstract}
In the Ouro Preto - MG region, there are several soap stone workshops where artesans create souvenirs and pans. This activity produces a large amount of residues which are discarded without any control. The objective of this work was to recover the talc mineral present in the size fraction below $74 \mu \mathrm{m}$ of these residues by froth flotation and chemical leaching with HCl. Among the flotation variables studied, only the percentage of solids and frother dosage proved to be significant for mass recovery and whiteness. This process produced a concentrate whose whiteness (ISO) was $68.56 \%$ and whose chemical composition meets paint and paper standards. After chemical leaching of the flotation concentrate, a whiteness of $84 \%$ was obtained and its chemical composition met the specifications of the plastic and anti-adherant plastic industries.
\end{abstract}

Keywords: Soapstone, talc, residues.

\section{Introdução}

O talco é o principal constituinte da pedra-sabão. Ele é usado em vasta gama 
de segmentos industriais devido às suas propriedades tecnológicas, tais como baixa dureza e maciez (1 na escala de Mohs), alta habilidade de cobertura, alto ponto de fusão, inércia química, baixa condutividade elétrica, alta capacidade de absorção de gordura, cor, óleos, resinas, baixa higroscopia e cor branca. É utilizado na indústria têxtil para impregnação de tecidos, na indústria de sabonetes, de pasta de dentes, na indústria de cosméticos de modo geral, na indústria de borracha (produção de tubos isolantes de fiações elétricas e na vulcanização), na indústria química como catalisador devido à sua grande área superficial entre outros itens. Quando puro, é utilizado na indústria farmacêutica. No caso de pureza variável, é uma importante matéria-prima para cerâmica elétrica (Kusvard, 1984). Na Tabela 1, estão apresentadas as especificações de talco para algumas aplicações industriais.

Por se tratar de um mineral com características hidrofóbicas, o talco pode ser flotado utilizando-se apenas espumante, no entanto, para uma melhora na cinética de flotação, faz-se necessária a utilização de coletores apolares como o querosene e o óleo de pinho como espumante. Esse sistema de reagentes tem sido a combinação mais adequada para a flotação de talcos, bem como a utilização de silicato de sódio como depressor (Leja, 1982). O processo de lixiviação também pode ser aplicado em etapas de purificação do talco e para um aumento conseqüente do seu teor. A

\begin{tabular}{|c|c|c|c|c|c|c|}
\hline Usos & Granul. (\#) & Composição Química & \begin{tabular}{|c|}
$\begin{array}{c}\text { Alvura } \\
\text { (ISO) }\end{array}$ \\
\end{tabular} & Mineralogia & Propriedades & Outros \\
\hline $\begin{array}{c}\text { Tintas: } \\
\text { Espalhador }\end{array}$ & $\begin{array}{c}100 \%<200 \\
96,5 \%<325\end{array}$ & $\begin{array}{c}\mathrm{SiO}_{2}+\mathrm{MgO} \geq 75 \% \\
\mathrm{Al}_{2} \mathrm{O}_{3} \leq 2 \% ; \mathrm{H}_{2} \mathrm{O}+\mathrm{M} . \mathrm{Vol} . \leq 1 \% ; \\
\mathrm{PPC} \leq 7 \%\end{array}$ & $65-87$ & $\begin{array}{l}\text { Partículas } \\
\text { lamelares }\end{array}$ & $\begin{array}{c}\text { Inércia química, } \\
\text { hidrofobicidade e } \\
\text { alvura }\end{array}$ & $\begin{array}{c}\text { Peso específico } \\
2,8 \text { a } 2,9\end{array}$ \\
\hline Pigmentos & $100 \%<325$ & $\begin{array}{c}\mathrm{MgO}-24 \text { a } 32 \% ; \mathrm{SiO}_{2}-50 \text { a } 65 \% \\
\mathrm{CaO} \leq 9 \% ; \mathrm{MgO}+\mathrm{SiO}_{2} \geq 88 \% \\
\mathrm{CO}_{2} \leq 1 \% ; \mathrm{PPC} \leq 7 \% \\
\mathrm{Al}_{2} \mathrm{O}_{3}+\mathrm{Fe}_{2} \mathrm{O}_{3} \leq 6 \% \\
\mathrm{H}_{2} \mathrm{O}+\mathrm{MV} \leq 1 \%\end{array}$ & $77-87$ & $\begin{array}{c}\text { Partículas } \\
\text { fibrosas } \\
\text { (tremolítico) }\end{array}$ & $\begin{array}{c}\text { Alvura, } \\
\text { inércia } \\
\text { química, } \\
\text { absorção de óleos } \\
27 \text { a } 31 \%\end{array}$ & $\begin{array}{c}\text { Peso específico } \\
2,8 \text { a } 2,9\end{array}$ \\
\hline Inseticida & $90 \%<325$ & $\mathrm{pH}-8$ & - & - & Inércia química & Adsorção \\
\hline Têxteis & $\begin{array}{c}\text { Sem resíduos } \\
\text { arenosos }\end{array}$ & - & - & $\begin{array}{l}\text { Sem minerais } \\
\text { de alta dureza }\end{array}$ & Alvura & - \\
\hline Papel (carga) & $95 \%<400$ & - & 54 a 75 & - & $\begin{array}{l}\text { Inércia química, } \\
\text { alvura }\end{array}$ & - \\
\hline $\begin{array}{l}\text { Plástico: } \\
\text { Carga e } \\
\text { reforço }\end{array}$ & $100 \%<200$ & \multirow[t]{2}{*}{ - } & \multirow{2}{*}{$\geq 74$} & \multirow{2}{*}{$\begin{array}{l}\text { Talco ou } \\
\text { pirofilita }\end{array}$} & \multirow{2}{*}{$\begin{array}{c}\text { Inércia química e } \\
\text { alvura }\end{array}$} & $\begin{array}{l}\text { Isento de } \\
\text { umidade }\end{array}$ \\
\hline $\begin{array}{c}\text { Anti- } \\
\text { aderente }\end{array}$ & $100 \%<325$ & & & & & Acidez baixa \\
\hline
\end{tabular}

\section{Materiais e métodos}

A amostra de resíduos de pedrasabão (aproximadamente $425 \mathrm{~kg}$ ), utilizada nesse trabalho, foi coletada em uma oficina de artesanato, localizada em Bandeiras - Santa Rita de Ouro Preto, que trabalha exclusivamente com rocha base dessa técnica é o fato de que o talco é inerte perante uma gama enorme de reagentes químicos e os compostos de ferro presentes podem ser reduzidos de $\mathrm{Fe}^{3+}$ para $\mathrm{Fe}^{2+}$ (Sarquis \& Gonzalez, 1998). Ahmed et al. (2007) obtiveram uma melhora no que se diz respeito à qualidade do talco pelo uso da lixiviação. Tal método consistiu na utilização de ácido clorídrico diluído $(10 \%)$ e uma temperatura de $60^{\circ} \mathrm{C}$ por um período de 30 minutos. Partindo de um concentrado de flotação de alvura (ISO) igual a 83,7\%, obteve-se um produto lixiviado de $88,5 \%$ de alvura (ISO).

$\mathrm{Na}$ região de Ouro Preto e Mariana - Minas Gerais, existem diversas pedreiras de pequeno porte, que explotam a pedra-sabão ou esteatito. A pedra-sabão é extraída em forma de blocos e exportada para a Europa, principalmente. Após o seu desdobramento em placas, a pedrasabão é empregadas em revestimentos internos de lareiras. Além da pedra-sabão, o talco, o qual ocorre em bolsões, é vendido in natura para a indústria cerâmica como um subproduto.

Lima et al. (2008) efetuaram caracterização mineralógica por difratometria de raios $\mathrm{X}$, microscopia ótica e microscopia eletrônica de varredura com sistema de análise de energia dispersiva de raios $\mathrm{X}$ (MEV/EDS) de amostras de pedra-sabão de cinco pedreiras da região de Bandeiras de Santa Rita de Ouro Preto. Os minerais identificados foram: talco, magnesita, dolomita, clorita, mica, tremolita/actinolita, serpentina, sulfetos (pirita, calcopirita, pentlandita), magnetita, hematita e outros.

Normalmente, a recuperação de rochas ornamentais, como é o caso da pedra-sabão da região de Ouro Preto, é de, no máximo, 30\%. Cerca de 70\% são de fragmentos menores, denominados de pontas e constituídos por uma rocha de qualidade inferior, que são vendidos para $\mathrm{o}$ artesanato, que a utiliza para fabricação de panelas, fôrmas de pizza, jarros e diversos objetos de adorno. Essa atividade é a principal fonte de renda da população das localidades e distritos da região como é o caso de Santa Rita de Ouro Preto, que fica a uma distância de $30 \mathrm{~km}$ de Ouro Preto, cujas coordenadas geográficas são: $20^{\circ} 32^{\prime} 09,3^{\prime \prime} \mathrm{S}$ e $43^{\circ} 33^{\prime} 01^{\prime \prime W}$. No entanto, somente cerca de $13 \%$ da rocha utilizada pelas oficinas de artesanato para fabricação de panelas é recuperada (Santos, 2009) ou seja, com essa atividade há um acréscimo na recuperação global de 9,1\% da rocha extraída nas pedreiras. Isso significa que $60 \%$ da mesma é descartada como resíduos, que são depositados sem nenhum controle nas proximidades das oficinas de artesanato.

Esse trabalho teve por objetivo recuperar o talco presente em resíduos de oficinas de artesanato para ser utilizado em alguma aplicação industrial, visando à diminuição do impacto ambiental e ao aumento da rentabilidade dos artesãos com a venda do talco contido e/ou recuperado desses resíduos.

Tabela 1

Especificações do talco para alguns usos industriais. Fonte: Modificado de Pontes \& Almeida (2005). daquela região, a qual foi previamente caracterizada por Lima et al. (2008).

Após homogeneização e quarteamento da amostra de resíduos no Laboratório de Tratamento de Minérios do DEMIN, foram retiradas alíquotas para caracterização granulométrica, química e mineralógica por difratometria de raios $\mathrm{X}$, sendo que os minerais identificados segundo Rodrigues (2010), foram talco $\left(\mathrm{Mg}_{3}\left(\mathrm{Si}_{2} \mathrm{O}_{5}\right)_{2}(\mathrm{OH})_{2}\right)$, magnesita $\left(\mathrm{MgCO}_{3}\right)$, dolomita $\left(\mathrm{CaMg}\left(\mathrm{CO}_{3}\right)_{2}\right)$, 
tremolita $\left(\mathrm{Ca}_{2} \mathrm{Mg}_{5} \mathrm{Si}_{8} \mathrm{O}_{22}(\mathrm{OH})_{2}\right)$, ilmentita $\left(\mathrm{FeTiO}_{3}\right)$ e nimita $(\mathrm{Ni}, \mathrm{Mg}, \mathrm{Al})_{6}(\mathrm{Si}$, $\left.\mathrm{Al})_{4}(\mathrm{OH})_{8}\right)$. Posteriormente, a referida amostra foi fracionada em peneira de $74 \mu \mathrm{m}$ de abertura, visando à purificação da fração acima de $74 \mu \mathrm{m}$ por métodos físicos (mesa e separação magnética de alta intensidade) efetuados por Rodrigues (2010) e flotação em bancada, utilizando uma célula mecânica de modelo CIMAQ com cuba de 2L do Laboratório de Flotação do DEMIN/UFOP para a fração abaixo de $74 \mu \mathrm{m}$. Os ensaios foram efetuados segundo planejamento estatístico de experimento fracionado (1/8) com réplica, sendo que as variáveis estudadas foram: dosagem de querosene - A (500 e $1000 \mathrm{~g} / \mathrm{t}$ ), adicionada como emulsão de $0,5 \% \mathrm{p} / \mathrm{v}$; metassilicato de sódio - B $(500$ e $1000 \mathrm{~g} / \mathrm{t})$, adicionado como solução de $0,5 \% \mathrm{p} / \mathrm{v}$; metil isobutil carbinol (MIBC) - C (75 e $150 \mathrm{~g} / \mathrm{t})$, adicionado como solução a 0,25\% p/v; pH - D (7 e 11), controlado pela adição de soluções de $\mathrm{HCl}$ ou $\mathrm{NaOH}$ a $1 \% \mathrm{p} / \mathrm{v}$; porcentagem de sólidos - E (10 e 20\%); rotação

Tabela 2

Análise granuloquímica da amostra global e da fração granulométrica abaixo de $74 \mu \mathrm{m}$ do resíduo de pedra-sabão da região de Bandeiras de Santa Rita de Ouro Preto.

\section{Resultados e discussão}

Pela análise dos resultados do planejamento fatorial de experimentos dos ensaios de flotação em bancada, utilizando o software MINITAB 15 - Ferramenta Gráfico de Pareto (nível

\section{Figura 1} Gráficos de cubo, porcentagem de sólidos vs dosagem de MIBC, para a Recuperação em Massa e Alvura dos ensaios de flotação. da célula - F (800 e 1000 RPM); tempo de condicionamento com o querosene - $G$ (5 e 10 min.); tempo de condicionamento com o metassilicato de sódio - H ( 5 e 10 min.); tempo de condicionamento com o espumante MIBC - I (5 e 10 min.). As variáveis-respostas analisadas foram a $\mathrm{Re}$ cuperação em Massa e a Alvura ISO do produto purificado, a qual foi determinada pelo Colorímetro Color Touch do Laboratório de Propriedades Interfaciais do DEMIN/UFOP, cuja metodologia foi descrita por Rodrigues (2010). Finalmente, efetuou-se alvejamento químico com ácido clorídrico de um concentrado de flotação (alvura ISO de 63,45\%), usando, também, planejamento estatístico de experimentos com réplica, cujas variáveis estudadas foram: concentração de $\mathrm{HCl}$ A (2 e 4M); temperatura - B $\left(50\right.$ e $\left.80^{\circ} \mathrm{C}\right)$ e tempo de lixiviação - C (30 e 60 min.). Nesse caso, foi analisada, como variávelresposta, somente a Alvura. As análises químicas das amostras e de todos os produtos da purificação foram efetuadas por plasma de acoplamento indutivo (Spec- tro modelo Ciros/CCD), exceto os teores de PPC e $\mathrm{SiO}_{2}$ (calculados por diferença (100 - PPC e teores de elementos maiores). Após os ensaios de lixiviação das amostras, foram retiradas alíquotas, que foram metalizadas e, posteriormente, analisadas no microscópio eletrônico de varredura (JOEL - Modelo 5510) do Laboratório de Microscopia e Microanálise do DEGEO, operado a $20 \mathrm{kV}$, visando, principalmente, a visualizar a forma das partículas de talco. Finalmente, foram efetuados ensaios de absorção de óleo, usando a norma técnica NBR 10417 e a determinação da densidade, usando, ainda, o picnômetro a gás (Ultrapicnometer 1000 do Laboratório de Propriedades Interfaciais do DEMIN/UFOP), com a amostra purificada.

Como pode ser observado pela $\mathrm{Ta}$ bela 2, as amostras in natura podem ser utilizadas somente para aplicação como carga em inseticidas, indústria têxtil, conforme especificações contidas na Tabela 1, necessitando de purificação para adequá-las a outras aplicações industriais.

\begin{tabular}{|c|c|c|c|c|c|c|c|c|c|c|}
\hline \multirow{3}{*}{$\begin{array}{c}\text { Fração } \\
\text { granulométrica } \\
(\mu \mathrm{m})\end{array}$} & \multirow{3}{*}{$\begin{array}{c}\text { Freq. } \\
\text { Simples } \\
(\%)\end{array}$} & \multicolumn{9}{|c|}{ Teor } \\
\hline & & \multicolumn{5}{|c|}{$\%$} & \multicolumn{4}{|c|}{ ppm } \\
\hline & & $\mathrm{Al}_{2} \mathrm{O}_{3}$ & $\mathrm{MgO}$ & $\mathrm{CaO}$ & $\mathrm{Fe}_{2} \mathrm{O}_{3}$ & $\mathrm{SiO}_{2}$ & As & $\mathrm{Cu}$ & $M n$ & $\mathrm{~Pb}$ \\
\hline+1168 & 14,00 & 2,35 & 27,90 & 0,05 & 5,20 & 58,47 & - & 11,8 & 261 & 10,1 \\
\hline$-1168+589$ & 10,40 & 2,42 & 28,20 & 0,05 & 5,31 & 58,04 & - & 12,50 & 270 & - \\
\hline$-589+208$ & 13,30 & 2,93 & 27,60 & 0,07 & 5,70 & 57,64 & 11,0 & 15,70 & 327 & - \\
\hline$-208+74$ & 11,80 & 2,25 & 27,30 & 0,09 & 5,46 & 58,95 & - & 23,60 & 439 & 11,50 \\
\hline$-74+105$ & 6,90 & 1,80 & 27,80 & 0,10 & 4,73 & 59,86 & - & 12,5 & 317 & 24,8 \\
\hline$-105+53$ & 7,60 & 1,92 & 28,3 & 0,06 & 4,47 & 59,71 & - & 9,78 & 241 & 26,5 \\
\hline$-53+37$ & 8,80 & 2,57 & 27,2 & 0,07 & 4,76 & 59,37 & 9,80 & 44,7 & 315 & 65,9 \\
\hline-37 & 27,30 & 2,11 & 28,0 & 0,07 & 4,51 & 59,48 & - & 10,9 & 261 & 49,6 \\
\hline Global $(-74 \mu \mathrm{m})$ & 50,60 & 2,18 & 27,88 & 0,07 & 4,58 & 59,55 & 1,70 & 16,83 & 275 & 45,58 \\
\hline Resíduo global & 100,00 & 2,30 & 27,80 & 0,07 & 4,99 & 58,91 & 2,32 & 16,30 & 298 & 25,82 \\
\hline
\end{tabular}

Alvura ISO da amostra global $(-74 \mu \mathrm{m})-51,30 \%$.

de confiança de 95\%), verificou-se que as variáveis: porcentagem de sólidos e dosagem de espumante (MIBC) tiveram influências significativas sobre a Recuperação em Massa do "talco" e somen- te a porcentagem de sólidos se mostrou significativa sobre a variável resposta Alvura. Na Figura 1, estão apresentados os gráficos de cubo dos valores médios dos resultados dos ensaios efetuados em

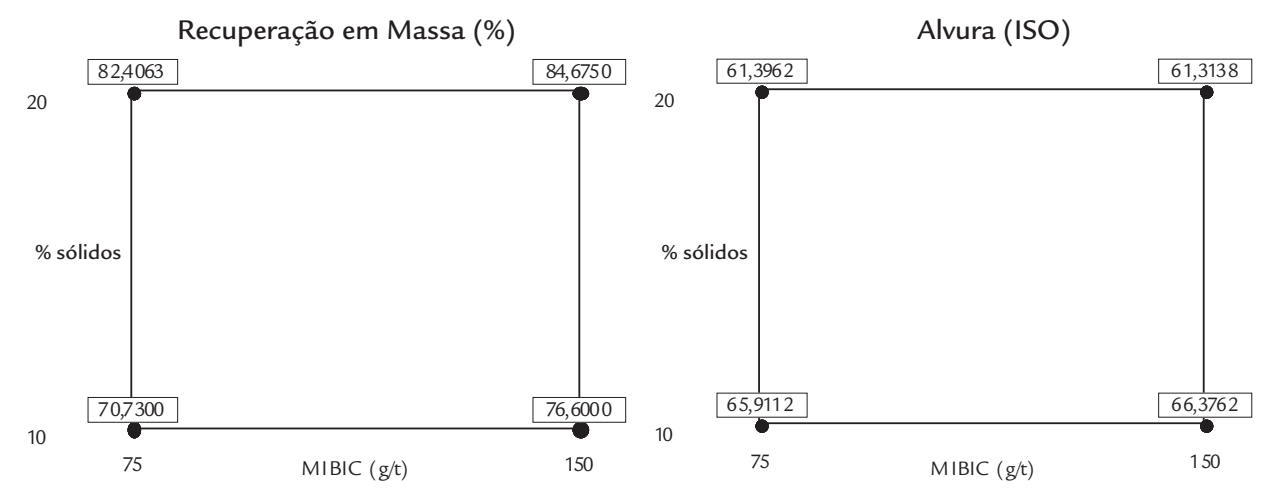

REM: R. Esc. Minas, Ouro Preto, 64(3), 353-357, jul. set. | 2011355 
relação às variáveis, que se mostraram significativas sobre as variáveis-respostas: Recuperação em Massa e Alvura ISO do produto purificado.

Utilizando-se da ferramenta estatística Resposta Otimizada do software MINITAB 15 para o alvo (Recuperação em Massa de 90\% e Alvura de $77 \%$ ), foi obtido o valor otimizado do modelo estatístico: Recuperação em Massa - 79,99\% e Alvura ISO - 64\% para dosagens de $500 \mathrm{~g} / \mathrm{t}$ de querosene e metassilicato de sódio, respectiva- mente, dosagem de $150 \mathrm{~g} / \mathrm{t}$ de MIBIC e porcentagem de sólidos de 14\%. De posse desses valores, efetuou-se o experimento em laboratório nas condições otimizadas (fixando as demais variáveis no valor mínimo, apresentadas anteriormente) acrescido de duas etapas cleaners, cuja recuperação em massa, alvura e composições químicas dos produtos purificados da etapa rougher e do segundo cleaner estão apresentadas na Tabela 3. Como pode ser observado, houve aumento de 5 pontos percentuais

\begin{tabular}{|c|c|c|c|c|}
\hline \multirow{6}{*}{$\begin{array}{l}\text { Teor } \\
(\mathrm{ppm})\end{array}$} & Constituinte & Alimentação & Rougher & $2^{\circ}$ Cleaner \\
\hline & As & 11,1 & - & - \\
\hline & $\mathrm{Cu}$ & 13,4 & 3,45 & 0,926 \\
\hline & $\mathrm{Ni}$ & 1956 & 2096 & 2126 \\
\hline & $\mathrm{Pb}$ & - & - & 8,80 \\
\hline & $\mathrm{Zn}$ & 85,8 & 67,0 & 64,4 \\
\hline \multirow{7}{*}{$\begin{array}{l}\text { Teor } \\
\text { (\%) }\end{array}$} & $\mathrm{Al}_{2} \mathrm{O}_{3}$ & 2,27 & 1,21 & 0,767 \\
\hline & $\mathrm{CaO}$ & 0,0580 & 0,0260 & 0,0180 \\
\hline & $\mathrm{Fe}_{2} \mathrm{O}_{3}$ & 4,70 & 4,00 & 3,57 \\
\hline & $\mathrm{MgO}$ & 27,7 & 28,3 & 27,7 \\
\hline & $\mathrm{TiO}_{2}$ & 0,0513 & 0,0383 & 0,0304 \\
\hline & PPC & 5,98 & 5,27 & 5,20 \\
\hline & $\mathrm{SiO}_{2}$ & 59,2 & 61,1 & 62,7 \\
\hline \multicolumn{2}{|c|}{ Recuperação em Massa (\%) } & 100 & 85,65 & 64,08 \\
\hline \multicolumn{2}{|c|}{ Alvura (ISO) } & 51,30 & 63,50 & 68,56 \\
\hline
\end{tabular}

Pela análise dos resultados do planejamento fatorial de experimentos dos ensaios de alvejamento químico do concentrado de flotação, utilizando-se o software MINITAB 15 - Ferramenta Gráfico de Pareto (nível de confiança de $95 \%$ ), verificou-se que todas as variáveis estudadas (dosagem de $\mathrm{HCl}$, temperatu-

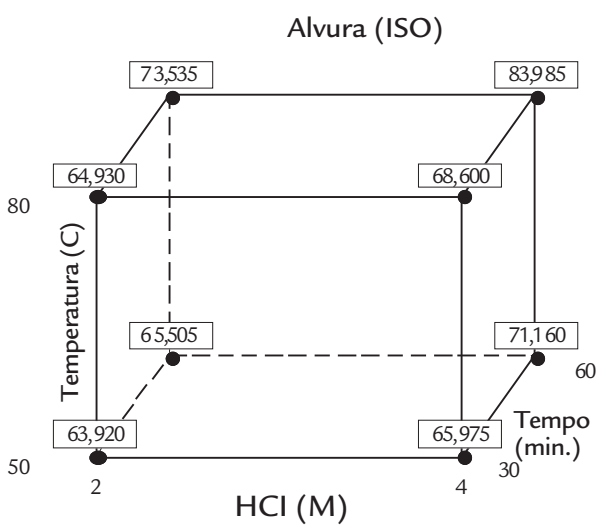

ra e tempo de lixiviação) foram significativas para a Alvura final do produto purificado. Na Figura 2, estão apresentados: o gráfico de cubo dos valores médios de Alvura dos ensaios efetuados, segundo o planejamento estatístico utilizado, e uma fotomicrografia do microscópio eletrônico de varredura característica do mate-

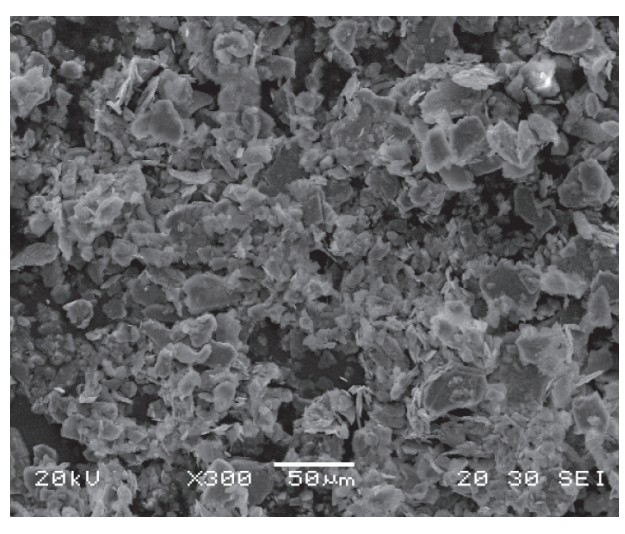

\begin{tabular}{c|c|c|c|c|c|c|c|c|c|c}
\hline \multirow{2}{*}{$\begin{array}{c}\text { Ensaio/ } \\
\text { Alvura }\end{array}$} & \multicolumn{9}{|c|}{ Ppm } & \multicolumn{7}{c}{ Teores } \\
\cline { 2 - 12 } & $\mathbf{C u}$ & $\mathbf{N i}$ & $\mathbf{Z n}$ & $\mathbf{A l}_{2} \mathbf{O}_{3}$ & $\mathbf{C a O}$ & $\mathbf{F e}_{2} \mathbf{O}_{3}$ & $\mathbf{M g O}$ & $\mathrm{TiO}_{2}$ & $\mathbf{P P C}$ & $\mathbf{S i O}_{2}$ \\
\hline $1 / 65,975$ & 3,62 & 1823 & 51,4 & 0,898 & 0,0193 & 2,61 & 28,8 & 0,0324 & 5,84 & 62,3 \\
\hline $2 / 64,93$ & 10,2 & 1929 & 60,5 & 0,929 & 0,0218 & 3,08 & 29,8 & 0,0326 & 5,13 & 61,0 \\
\hline $3 / 83,985$ & 6,60 & 1916 & 58,9 & 0,951 & 0,0207 & 3,42 & 30,0 & 0,0343 & 5,21 & 60,3 \\
\hline $4 / 63,92$ & 4,65 & 1923 & 56,8 & 0,969 & 0,0287 & 3,23 & 29,9 & 0,0350 & 5,28 & 60,6 \\
\hline $5 / 81,16$ & 3,74 & 1900 & 56,1 & 0,823 & 0,0255 & 3,04 & 30,2 & 0,0335 & 4,44 & 61,7 \\
\hline $6 / 68,60$ & 9,18 & 1940 & 58,1 & 0,978 & 0,0250 & 3,28 & 30,0 & 0,0349 & 4,90 & 60,8 \\
\hline $7 / 73,535$ & 6,70 & 1929 & 59,3 & 0,977 & 0,0327 & 3,43 & 29,5 & 0,0352 & 4,97 & 61,1 \\
\hline $8 / 65,505$ & 4,49 & 1936 & 59,7 & 0,913 & 0,0350 & 3,29 & 30,9 & 0,0339 & 5,16 & 59,9 \\
\hline
\end{tabular}

na Alvura após duas etapas cleaners. No entanto, a Recuperação em Massa caiu $21,6 \%$.

De uma maneira geral, observouse que os s produtos purificados por flotação em bancada dos resíduos de pedra-sabão abaixo de $74 \mu \mathrm{m}$ (200\#) atenderam às especificações em termos de alvura e composição química para a sua utilização como matéria-prima para as indústrias de inseticidas, têxteis, fertilizantes, papel (carga), tintas (espalhador) (veja especificações na Tabela 1).

Tabela 3

Composição química da amostra abaixo de $74 \mu \mathrm{m}$ in natura e dos produtos purificados por flotação na condição otimizada.

rial lixiviado. Os resultados médios das análises químicas e alvura dos produtos do alvejamento químico com $\mathrm{HCl}$ estão apresentados na Tabela 4.

Pela Figura 2 (diagrama de cubos), foi obtida alvura ISO de, aproximadamente, 84\%. Rodrigues (2010), após lixiviação de amostra de um concentrado de

Figura 2

Gráfico de cubo para os resultados da Alvura referente aos ensaios de alvejamento com ácido clorídrico $(\mathrm{HCl})$ e fotomicrografia do produto lixiviado (imagem de elétrons secundários, aumento de 300 vezes).

Tabela 4

Resultados médios de alvura e teores dos produtos do alvejamento químico com ácido clorídrico $(\mathrm{HCl})$. 
flotação desse mesmo material com ditionito de sódio $\left(\mathrm{Na}_{2} \mathrm{~S}_{2} \mathrm{O}_{4}\right)$, obteve alvura final de 65,83\%. O melhor desempenho do $\mathrm{HCl}$ em relação ao ditionito de sódio na purificação desse material ocorreu devido à dissolução dos carbonatos presentes na amostra pelo $\mathrm{HCl}$ (Ahmed et al., 2007), o que ocasionou um valor de alvura final muito superior àquele obtido com o ditionito de sódio.

Como pode ser observado na Tabela 4, não foi identificado os elementos químicos $\mathrm{As}$ e $\mathrm{Pb}$, que estavam abaixo do limite de detecção do equipamento

\section{Conclusões}

O resíduo de pedra-sabão (amostra global) e a fração granulométrica abaixo de $74 \mu \mathrm{m}$ possuem especificações químicas para ser usado na fabricação de inseticida (veículo).

A otimização dos ensaios de flota-

\section{Agradecimentos}

Os autores agradecem à FAPEMIG pelo financiamento do projeto, o qual possibilitou a compra de equipamentos do Laboratório de Propriedades Inter-

\section{Referências bibliográficas}

utilizado (9,45 e 8,30, respectivamente para o As e $\mathrm{Pb}$ ). As reduções dos teores de $\mathrm{MgO}$ e $\mathrm{CaO}$ estão associadas à dissolução dos carbonatos (dolomita e magnesita), presentes no material. Os teores de $\mathrm{F}_{2} \mathrm{O}_{3}$ estão associados ao $\mathrm{Fe}$ proveniente dos minerais pentlandita, calcopirita e ilmenita, presentes na amostra estudada.

Conforme pode ser observado na Figura 2, as partículas de talco são lamelares. Pela comparação dos valores obtidos na Tabela 4 com as especificações apresentadas na Tabela 1 , verificou-se

ção $(500 \mathrm{~g} / \mathrm{t}$ de querosene e metassilicato de sódio de $150 \mathrm{~g} / \mathrm{t}$ de MIBC), seguida de duas etapas cleaners, possibilitou a obtenção de um produto purificado com especificações para tintas (espalhador) e papel (carga).

faciais do DEMIN/UFOP, que são de extrema importância para o desenvolvimento de inúmeros trabalhos de pesquisa da área de Tratamento de Minérios que, após o alvejamento químico com $\mathrm{HCl}$, foram obtidas especificações para as indústrias de plásticos (carga e reforço) e plásticos antiaderente.

Os valores CIE, ligtness $\left(\mathrm{L}^{*}\right)$ redness $\left(a^{*}\right)$ e yellowness $\left(b^{*}\right)$ da amostra de concentrado do ensaio 3 (Tabela 4), cuja alvura aproximada era de $84 \%$, foram os seguintes: $L^{*}=94,62, a^{*}=-0,11$ $\mathrm{e} \mathrm{b}^{*}=2,22$.

A absorção de óleo do produto lixiviado foi de $28,5 \%$ e a densidade foi de 2,9, valores que estão dentro dos limites apresentados na Tabela 1.

$\mathrm{O}$ alvejamento químico com $\mathrm{HCl}$ adequou o produto alvejado às industriais de plásticos (carga e reforço) e plásticos antiaderentes, além das especificações já citadas anteriormente.

do Programa de Pós-Graduação em Engenharia Mineral - PPGEM e ao CNPq pela concessão da bolsa de mestrado para um dos autores.

ABNT - Associação Brasileira de Normas Técnicas. Pigmentos e cargas - determinação do valor de absorção de óleo (NBR 10417). Rio de Janeiro. 1988. 4p.

AHMED, M. M., IBRAHIM, G. A., HASSAN, M. M.A. Improvement of Egyptian talc quality for industrial uses by flotation process and leaching. International Journal of Mineral Processing, v. 83, p.132-145, 2007.

KUZVART, M. Industrial Minerals and Rocks. Amsterdam: Elsevier, 1984. 254p.

LEJA, J. Surface Chemistry of Froth Flotation. 2 ed. New York: Plenum Publishers, 1982. $757 \mathrm{p}$.

LIMA, R. M. F., SILVA, F. B. R., BRAGA, D. M. Caracterização tecnológica de pedra-sabão de pedreiras e de oficinas de artesanato em pedra-sabão de Ouro Preto, visando a um melhor aproveitamento econômico. Ouro Preto: DEMIN। UFOP, 2008. 101p. (Relatório Técnico).

PONTES, I. F., ALMEIDA, S. L. M. Talco. In: Rochas e minerais industriais usos e especificações. LUZ, A. B., LINS, F. F. (Ed.). Rio de Janeiro: CETEM/MCT. 2005. p. 607-628.

SANTOS, R. C. P. Análise dos entraves para a criação de um arranjo produtivo local (APL) de base mineral da pedra-sabão na região de Ouro Preto, Minas Gerais. Ouro Preto: Programa de Pós-Graduação em Engenharia Mineral - Universidade Federal de Ouro Preto, 2009. 79p. (Dissertação de Mestrado).

SARQUIS, P.E., GONZALEZ, M. Limits of the use of industrial talc- the carbonate effect. Minerals Engineering, v.11, n. 7, p. 657-660, 1998.

RODRIGUES, M. L.M. Caracterização tecnológica de resíduos de pedra-sabão de oficinas de artesanato em pedra-sabão da região de Ouro Preto. Ouro Preto: Programa de Pós-Graduação em Engenharia Mineral, Universidade Federal de Ouro Preto, 2010. 137p. (Dissertação de Mestrado).

Artigo recebido em 28 de abril de 2010. Aprovado em 19 de maio de 2011. 